\title{
Demographic And Clinical Characteristics Of
}

\section{Patients Prescribed Proprotein Convertase Subtilisin/ kexin Type 9 Inhibitor Therapy And Patients Whose Current Lipid-Lowering Therapy Was Modified}

This article was published in the following Dove Press journal:

Therapeutics and Clinical Risk Management

Seth J Baum ${ }^{1,2}$
Rolin L Wade ${ }^{3}$
Pin Xiang
Jorge Arellano
Cesar Cerezo Olmos
Sasikiran Nunna
${ }^{5}$
Chi-Chang Chen ${ }^{6}$
Cathryn M Carter
Nihar R Desai
'
'Department of Integrated Medical
Sciences, Charles E Schmidt College of
Medicine, Florida Atlantic University,
Boca Raton, FL, USA; ${ }^{2}$ Preventive
Cardiology Inc, Boca Raton, FL, USA;
${ }^{3}$ Medical and Scientific Services, IQVIA,
Plymouth Meeting, PA, USA; ${ }^{4}$ Global
Health Economics, Amgen Inc, Thousand
Oaks, CA, USA; ${ }^{5}$ US Medical, Amgen Inc,
Thousand Oaks, CA, USA; ${ }^{6}$ Real-World
Evidence Solutions, IQVIA, Plymouth
Meeting, PA, USA; ${ }^{7}$ Global Publications,
Amgen Inc, Thousand Oaks, CA, USA;
${ }^{8}$ Section of Cardiovascular Medicine,
Center for Outcomes Research and
Evaluation, Yale School of Medicine, New
Haven, CT, USA

Haven, CT, USA
Purpose: Our objective was to describe the demographic and clinical characteristics of real-world patients in the US with elevated low-density lipoprotein cholesterol (LDL-C) whose lipid-lowering therapy (LLT) - both proprotein convertase subtilisin/kexin type 9 (PCSK9) inhibitor and non-PCSK9 inhibitor - was actively modified.

Methods: This retrospective cohort study used linked laboratory (Prognos), pharmacy (IMS Formulary Impact Analyzer), and medical claims (IQVIA Dx/LRx or PharMetrics Plus) data. PCSK9 inhibitor-prescribed patients with LDL-C $\geq 70 \mathrm{mg} / \mathrm{dL}$ (multiply by 0.02586 for $\mathrm{mmol} / \mathrm{L}$ ) at the time of prescription were matched by LDL-C test date to patients whose non-PCSK9 inhibitor therapy was modified by intensifying statin therapy, switching statins without intensification, or augmenting with ezetimibe ( $\mathrm{N}=12,345$ in each cohort). Baseline demographics, use of LLT, LDL-C values, atherosclerotic cardiovascular disease (ASCVD) diagnoses and cardiovascular comorbidities, and occurrence of major adverse cardiovascular events (MACE) were assessed during the 2-year pre-index period.

Results: Mean age was 66.2 years in the PCSK9 inhibitor cohort and 64.1 years in the cohort whose LLT regimen was otherwise modified. Respectively, mean baseline LDL-C values were 150 and $121 \mathrm{mg} / \mathrm{dL} ; 60.3 \%$ and $39.0 \%$ of patients had ASCVD diagnoses, and 9.6\% and $5.1 \%$ had experienced a recent MACE. Prevalence of ASCVD diagnoses in the PCSK9 inhibitor and modified non-PCSK9 inhibitor cohorts, respectively, was $15.5 \%$ vs $9.1 \%$ for acute coronary syndrome, $20.7 \%$ vs $8.7 \%$ for coronary revascularization, and $22.2 \%$ vs $5.1 \%$ for possible familial hypercholesterolemia. In addition, $19.8 \%$ of patients in the PCSK9 inhibitor cohort were receiving both statins and ezetimibe vs $5.0 \%$ in the modified LLT cohort.

Conclusion: Physicians are prescribing PCSK9 inhibitor therapy to patients with markedly elevated LDL-C levels who also have comorbid risk factors for adverse cardiovascular events. These results may be of interest to payers and policymakers involved in devising access strategies for PCSK9 inhibitors.

Keywords: cardiovascular risk, lipid-lowering therapy, low-density lipoprotein, PCSK9 inhibitor, real-world treatment patterns

\section{Introduction}

In early 2018, it was estimated that in that year approximately 720,000 Americans would be hospitalized with a first myocardial infarction (MI) or would die because of coronary heart disease, and approximately 335,000 survivors would have a 
recurrent event. ${ }^{1}$ Similarly, an estimated 795,000 people experience a new $(610,000)$ or recurrent $(185,000)$ stroke annually; $87 \%$ of these events are ischemic in origin. ${ }^{1}$ Coronary heart disease is responsible for $43.8 \%$ of cardiovascular (CV)-related deaths in the US, followed by stroke (16.8\%) and other cardiovascular diseases (CVDs; $17.9 \%) .{ }^{1}$ In 2016 , approximately 544,800 people died of ischemic heart disease and 113,000 died of stroke. ${ }^{2}$ These premature deaths were associated with 7,605,300 and $1,139,800$ years of life lost, respectively. In addition, the economic burden of CVD is substantial and increasing. The combined direct and indirect cost burden of CVD in 2016 was $\$ 555$ billion (direct medical expenses, \$318 billion; indirect costs, $\$ 237$ billion). ${ }^{3}$ By $2035,45.1 \%$ of adults in the US are projected to have some form of CVD, and this burden is expected to cost $\$ 1.1$ trillion (direct, $\$ 749$ billion; indirect, $\$ 368$ billion).

Low-density lipoprotein cholesterol (LDL-C) plays a central role in the pathogenesis of atherosclerotic cardiovascular disease (ASCVD), and this relationship is both dose- and time-dependent. ${ }^{4,5}$ Although statins remain the cornerstone of lipid-lowering therapy (LLT), most patients with ASCVD do not achieve treatment goals with statins alone. ${ }^{6,7}$ The proprotein convertase subtilisin/kexin type 9 (PCSK9) inhibitor monoclonal antibodies represent an additional option for lowering of LDL-C levels in patients with ASCVD for whom maximally tolerated statin therapy, with or without augmentation with ezetimibe, is inadequate. ${ }^{8-10}$ For the first time, PCSK9 inhibitor therapies have been included, as Class IIa evidence for very high-risk patients with ASCVD, in the 2018 American College of Cardiology/American Heart Association (ACC/AHA) clinical practice guideline for the management of blood cholesterol. ${ }^{10}$ The 2018 ACC/AHA cholesterol guideline also introduces an LDL-C threshold of $\geq 70$ $\mathrm{mg} / \mathrm{dL}(\geq 1.8 \mathrm{mmol} / \mathrm{L}$; multiply $\mathrm{mg} / \mathrm{dL}$ by 0.02586 for $\mathrm{mmol} / \mathrm{L}$ ) as a trigger for treatment decisions in patients with very-high-risk ASCVD already receiving maximally tolerated statin and/or ezetimibe therapy.

Although early barriers to access and reimbursement for PCSK9 inhibitor therapy seem to be decreasing, ${ }^{11}$ overall approval rates for PCSK9 inhibitors were $<50 \%$ between July 2015 and August 2016. ${ }^{12,13}$ A previous analysis of early adopters of PCSK9 inhibitor therapy in the US found that patients treated with PCSK9 inhibitors had higher CV risk in terms of LDL-C levels, CV comorbidities, statin intolerance, and intensity of LLT compared with patients treated with LLTs other than PCSK9 inhibitors. ${ }^{14}$ We aimed to describe the CV risk profiles of two distinct cohorts of patients - those prescribed PCSK9 inhibitor therapy and those whose non-PCSK9 inhibitor LLT had been recently modified (ie, intensified, switched, or augmented with ezetimibe). Characterization of these two profiles should answer current questions about the clinical appropriateness of PCSK9 inhibitor prescribing patterns in real-world clinical practice in the US.

\section{Methods}

\section{Study Design And Patient Population}

This retrospective cohort study used linked pharmacy adjudication status (IQVIA Formulary Impact Analyzer [FIA]; IQVIA, Plymouth Meeting, PA, USA), medical and prescription claims (IQVIA Dx/LRx or PharMetrics Plus $[\mathrm{P}+]$ ), and laboratory data (Prognos, New York, NY, USA) from July 1, 2013, to December 31, 2017 (Figure 1). The FIA is a nationally representative transactional pharmacy claims database that captures complete transaction records for prescription transactions (approval, abandonment, and rejection). The LRx database contains information related to adjudicated dispensed prescriptions, which are sourced from retail, mail, long-term care, and specialty pharmacies. This database is sourced from pharmacies and represents $>90 \%$ of all outpatient prescriptions dispensed in the US. The Dx database contains unadjudicated medical claims from office-based physicians, ambulatory facilities, and general health care sites. Dx data are supplemented with institutional claims, including claims from hospital-based physicians. This database is sourced from "clearing houses," which are also referred to as "switches." These data represent approximately $75 \%$ of all the physicians in the American Medical Association database and capture $>1.3$ billion claims per year. The $\mathrm{P}+$ database contains adjudicated medical and pharmacy claims of patients in the US and is sourced from payers. This database contains standard fields such as inpatient and outpatient diagnoses and procedures, and retail and mail order prescription records; $\mathrm{P}+$ has detailed information on the pharmacy and medical benefit (copayment, deductible), the inpatient stay (admission type and source, discharge status), and provider details (specialty, provider ID). The database includes $>150$ million unique individuals, with approximately 40 million active patients in the database in 2017.

Patients in all IQVIA databases and the Prognos LDL-C dataset were linked at a patient level using a deterministic encryption method that complies with Health Insurance Portability and Accountability Act (HIPAA) regulations. The 


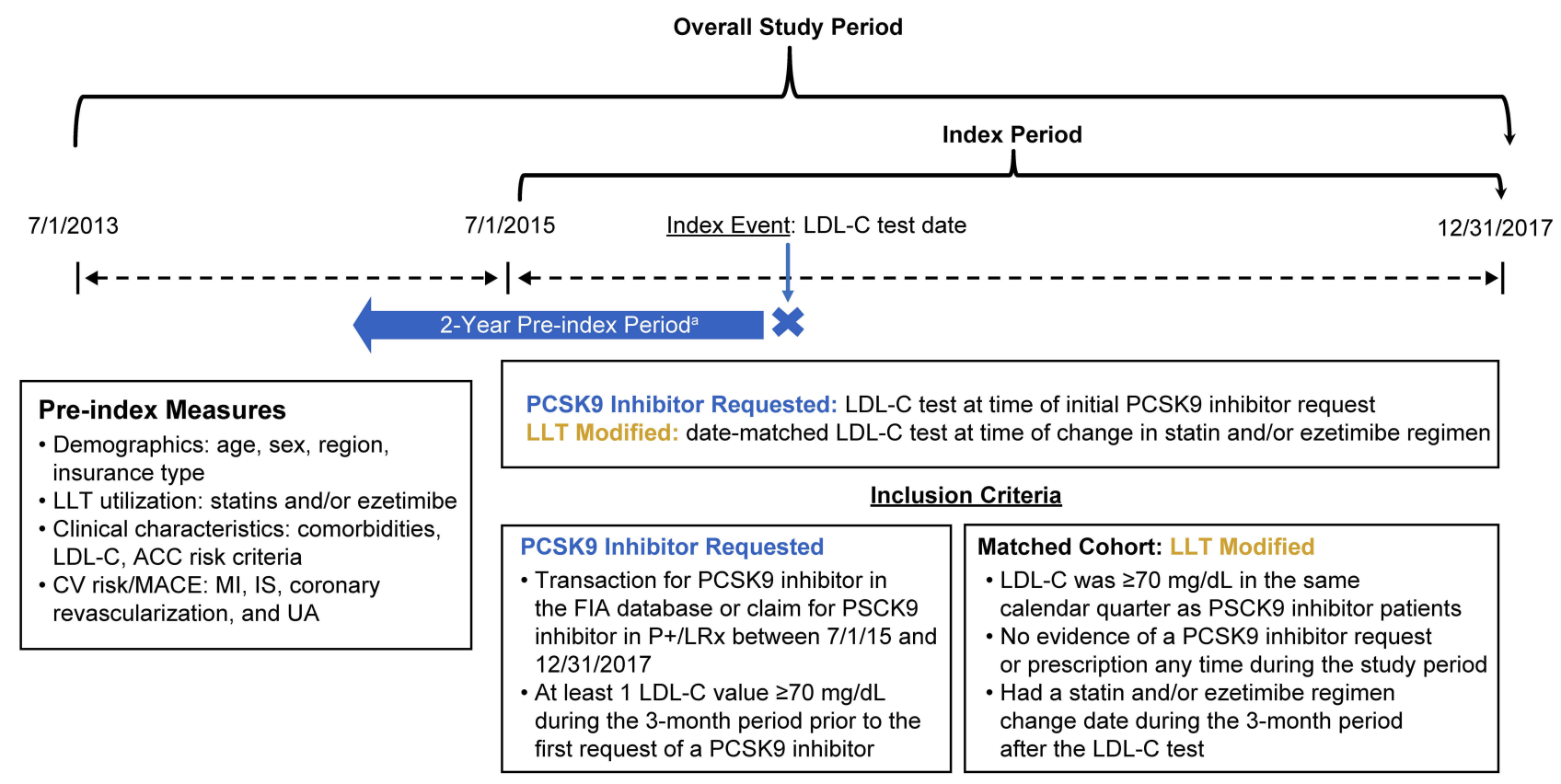

Figure I Study design and patient population: study period from $7 / 1 / 2013$ to $12 / 31 / 2017$.

Notes: ${ }^{a}$ Floating 2-year index period that changed with the exact index date for each patient. For example, if a patient was indexed on $7 / 1 / 2016$, the 2-year pre-index period would be from $7 / 1 / 2014$ to $7 / 1 / 2016$. Only patients indexed exactly on the first day of the indexing period (7/I/2015) would have a pre-index period from $7 / 1 / 2013$ to $7 / 1 / 2015$.

Abbreviations: ACC, American College of Cardiology; ASCVD, atherosclerotic CV disease; CV, cardiovascular; FIA, Formulary Impact Analyzer; IS, ischemic stroke; LDL-C, lowdensity lipoprotein cholesterol; LLT, lipid-lowering therapy; MACE, major adverse CV events; MI, myocardial infarction; P+, PharMetrics Plus; PCSK9, proprotein convertase subtilisin/kexin type 9; UA, unstable angina.

PCSK9 inhibitor cohort included patients who submitted a prescription for PCSK9 inhibitor therapy. The cohort whose existing statin or ezetimibe regimen was modified included intensification of the current statin regimen by either increasing the dose of the current statin or switching statins at a higher intensity; by switching to a different statin, without an increase in statin intensity; or by augmenting statin therapy with ezetimibe. Supplementary Table S1 provides the statin intensity rules. Patients who switched statins without intensification were included in the modified non-PCSK9 inhibitor cohort, because studies have shown substantial LDL-C lowering among statin-treated patients who switched to a different statin at the same intensity. ${ }^{15,16}$ Included patients were $\geq 18$ years old, had a pre-index LDL-C level $\geq 70 \mathrm{mg} / \mathrm{dL}$ at the time of prescription for PCSK9 inhibitor therapy or modification of other LLT, and had high-quality linkable data in the IQVIA claims databases (Figure 1; Supplementary Table S2). Patients in the modified LLT cohort were matched 1:1 to patients in the PCSK9 inhibitor cohort by the LDL test date (within the same calendar quarter; $\mathrm{N}=12,345$ per cohort). The index event was the LDL-C test at the time of initial PCSK9 inhibitor request (PCSK9 inhibitor cohort) or the date-matched LDL-C test at the time of the change in statin and/or ezetimibe regimen (modified LLT cohort).

\section{Pre-Index Measures And Index Event}

All variables were measured during a 2-year baseline period prior to the index event. The index period was from July 1, 2015, to December 31, 2017, and the index event for each patient was the qualifying LDL-C test date (LDL-C $\geq 70 \mathrm{mg} / \mathrm{dL}$ ).

Pre-index measures included demographics (age, sex, geographic region, insurance type), clinical characteristics (ASCVD diagnoses, CV comorbidities, LDL-C values), LLT utilization, and CV risk as measured by occurrence of recent major adverse $\mathrm{CV}$ events (MACE). MACE included events of MI, ischemic stroke (IS), coronary revascularization (coronary artery bypass graft or percutaneous coronary intervention), and unstable angina (UA). ASCVD diagnoses, $\mathrm{CV}$ comorbidities and risk factors, and MACE were identified using International Classification of Diseases (ICD)-9/ ICD-10 diagnosis codes. Baseline demographic and clinical measures were summarized descriptively. This study was not designed to make statistical comparisons between cohorts and did not assess post-index outcomes.

\section{Ethics}

This study complied with all applicable laws regarding subject privacy, using HIPAA-compliant de-identified 
retrospective data sources. No direct subject contact or primary collection of individual human subject data occurred. Study results were in tabular form and aggregate analyses that omitted subject identification; therefore, informed consent, ethics committee approval, and institutional review board approval were not required.

\section{Results}

\section{Demographics And Clinical}

\section{Characteristics}

Mean age was 66.2 years in the PCSK9 inhibitor cohort and 64.1 years in the cohort whose LLT regimen was modified; $58.4 \%$ and $49.0 \%$ of patients, respectively, were $\geq 65$ years of age (Table 1). Regarding ezetimibe use, one in five patients (19.8\%) in the PCSK9 inhibitor cohort and one in $20(5.0 \%)$ in the cohort whose statin and/or ezetimibe regimen was modified were receiving both statins and ezetimibe.

For the non-PCSK9 inhibitor cohort, there were no overt or unexpected directional differences in the constellation of demographic or clinical characteristics among the subgroups whose statin regimen was intensified, switched, or augmented with ezetimibe (Supplementary Tables S3 and $\underline{\mathrm{S} 4}$ ).

\section{Baseline LDL-C Values}

Mean LDL-C levels at index were $150 \mathrm{mg} / \mathrm{dL}$ (SD, 48.9 $\mathrm{mg} / \mathrm{dL}$ ) in the PCSK 9 inhibitor cohort and $121 \mathrm{mg} / \mathrm{dL}$ $(\mathrm{SD}, 37.5 \mathrm{mg} / \mathrm{dL})$ in the cohort whose non-PCSK9 inhibitor regimen was modified (Figure 2). Baseline LDL-C levels were $\geq 130 \mathrm{mg} / \mathrm{dL}$ in $62.7 \%$ of patients in the PCSK9 inhibitor cohort and $36.2 \%$ in the modified LLT cohort; LDL-C levels were $\geq 100 \mathrm{mg} / \mathrm{dL}$ in $85.4 \%$ and $66.1 \%$ of patients, respectively. In the subset of patients of the modified LLT cohort who had their statin regimen augmented with ezetimibe $(\mathrm{n}=764)$, mean LDL-C was $115 \mathrm{mg} / \mathrm{dL}$ (SD, $38.2 \mathrm{mg} / \mathrm{dL}$ ), and $57.5 \%$ of patients had LDL-C levels $\geq 100 \mathrm{mg} / \mathrm{dL}$ (Supplementary Table S4).

\section{ASCVD Diagnoses And Comorbidities}

The percentages of patients with ASCVD diagnoses in the PCSK9 inhibitor and modified non-PCSK9 inhibitor cohorts, including any ASCVD diagnosis, acute coronary syndrome (defined by MI or UA), stroke (IS or transient ischemic attack), coronary revascularization, and possible familial hypercholesterolemia (defined as LDL-C $\geq 190 \mathrm{mg} / \mathrm{dL}$ any time during the baseline period), are summarized in Figure 3A. A history of ASCVD was present for $60.2 \%$
Table I Baseline Demographic And Clinical Characteristics: Matched Cohorts

\begin{tabular}{|c|c|c|}
\hline & $\begin{array}{l}\text { PCSK9 } \\
\text { Inhibitor } \\
\text { Requested } \\
(\mathrm{N}=\mid 2,345)\end{array}$ & $\begin{array}{l}\text { LLT Regimen } \\
\text { Intensified/ } \\
\text { Switched/ } \\
\text { Augmented } \\
(\mathrm{N}=\mid 2,345)\end{array}$ \\
\hline \multicolumn{3}{|l|}{ Demographics } \\
\hline $\begin{array}{l}\text { Age, years } \\
\text { Mean (SD) } \\
\text { Median }\end{array}$ & $\begin{array}{l}66.2(9.8) \\
67\end{array}$ & $\begin{array}{l}64.1(11.0) \\
64\end{array}$ \\
\hline $\begin{array}{l}\text { Age group, n }(\%) \\
\text { 18-34 years } \\
35-44 \text { years } \\
45-54 \text { years } \\
55-64 \text { years } \\
\geq 65 \text { years }\end{array}$ & $\begin{array}{l}30(0.2) \\
206(1.7) \\
1,242(10.1) \\
3,654(29.6) \\
7,213(58.4)\end{array}$ & $\begin{array}{l}83(0.7) \\
419(3.4) \\
1,822(14.8) \\
3,97 \mid(32.2) \\
6,050(49.0)\end{array}$ \\
\hline $\begin{array}{l}\text { Sex, n (\%) } \\
\quad \text { Men } \\
\text { Women }\end{array}$ & $\begin{array}{l}5,7 \mid 2(46.3) \\
6,633(53.7)\end{array}$ & $\begin{array}{l}5,834(47.3) \\
6,5 \text { II (52.7) }\end{array}$ \\
\hline $\begin{array}{l}\text { Geographic region, n (\%) } \\
\text { Northeast } \\
\text { Midwest } \\
\text { South } \\
\text { West }\end{array}$ & $\begin{array}{l}2,469(20.0) \\
937(7.6) \\
7,245(58.7) \\
1,694(13.7)\end{array}$ & $\begin{array}{l}2,920(23.7) \\
1,109(9.0) \\
6,763(54.8) \\
1,553(12.6)\end{array}$ \\
\hline $\begin{array}{l}\text { Payer type, n (\%) } \\
\text { Commercial } \\
\text { Medicare } \\
\text { Other }\end{array}$ & $\begin{array}{l}7,348(59.5) \\
4,935(40.0) \\
62(0.5)\end{array}$ & $\begin{array}{l}6,969(56.5) \\
4,774(38.7) \\
602(4.9)\end{array}$ \\
\hline Clinical characteristics & & \\
\hline $\begin{array}{l}\mathrm{CCl} \\
\text { Mean (SD) } \\
\text { Median } \\
\geq 3 \text { comorbidities, n (\%) }\end{array}$ & $\begin{array}{l}2.5(2.5) \\
2 \\
4,894(39.6)\end{array}$ & $\begin{array}{l}2.6(2.8) \\
2 \\
4,868(39.4)\end{array}$ \\
\hline $\begin{array}{l}\text { LLT use, } n(\%) \\
\text { Statin only } \\
\text { High intensity } \\
\text { Medium intensity } \\
\text { Low intensity } \\
\text { Statin + ezetimibe } \\
\text { High intensity } \\
\text { Medium intensity } \\
\text { Low intensity }\end{array}$ & $\begin{array}{l}5,525(44.8) \\
1,738(14.1) \\
2,962(24.0) \\
825(6.7) \\
2,444(19.8) \\
955(7.7) \\
1,110(9.0) \\
379(3.1)\end{array}$ & $\begin{array}{l}11,734(95.0) \\
1,856(15.0) \\
7,717(62.5) \\
2,161(17.5) \\
611(5.0) \\
128(1.0) \\
403(3.3) \\
80(0.7)\end{array}$ \\
\hline
\end{tabular}

Abbreviations: CCl, Charlson Comorbidity Index; LLT, lipid-lowering therapy; PCSK9, proprotein convertase subtilisin/kexin type 9.

and $39.0 \%$ of patients, respectively. In the subgroup for which the LLT regimen was augmented with ezetimibe, $52.4 \%$ of patients had ASCVD (Supplementary Table S4). 


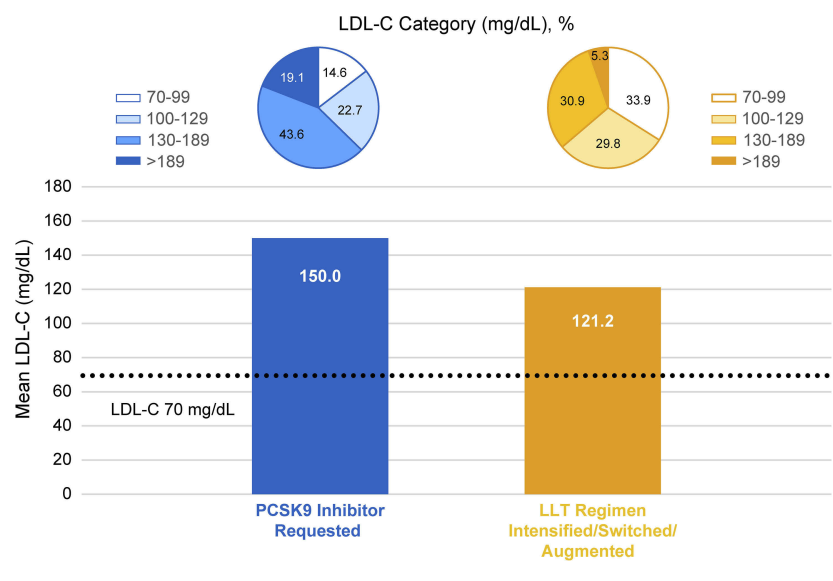

Figure 2 Mean baseline LDL-C value at index.

Note: To convert from $\mathrm{mg} / \mathrm{dL}$ to $\mathrm{mmol} / \mathrm{L}$, multiply by 0.02586 .

Abbreviations: LDL-C, low-density lipoprotein cholesterol; LLT, lipid-lowering therapy; PCSK9, proprotein convertase subtilisin/kexin type 9.

Prevalence of ASCVD diagnoses in the PCSK9 inhibitor and modified non-PCSK9 inhibitor cohorts, respectively, was $15.5 \%$ and $9.1 \%$ for acute coronary syndrome, $8.2 \%$ and $8.0 \%$ for stroke, $20.7 \%$ and $8.7 \%$ for coronary revascularization, and $22.2 \%$ and $5.1 \%$ for possible familial hypercholesterolemia. Type 2 diabetes and hypertension were the most common high-risk $\mathrm{CV}$ comorbidities in both groups (Figure 3B).

\section{Risk}

Nearly $10 \%$ of patients had at least one acute MACE (MI, IS, UA, or coronary revascularization) in the 2 years prior to their PCSK9 inhibitor prescription (Figure 4). In the cohort whose statin and/or ezetimibe LLT regimen was modified, $5.1 \%$ of patients had at least one acute MACE. Coronary revascularization was the most common MACE in both groups $(7.4 \%$ in the PCSK9 inhibitor cohort vs $3.0 \%$ in the modified non-PCSK 9 inhibitor cohort).

\section{Discussion}

This real-world analysis characterizing the $\mathrm{CV}$ risk of patients receiving a prescription for a PCSK9 inhibitor from July 2015 through 2017, prior to release of the 2018 ACC/AHA guideline, ${ }^{10}$ demonstrates that physicians were prescribing PCSK9 inhibitor therapy to patients with LDL-C values more than double the currently recommended target of $<70 \mathrm{mg} / \mathrm{dL}$ for patients with very high-risk ASCVD. In addition to markedly elevated LDL-C, many patients also had clinical ASCVD, high-risk comorbidities, and/or a recent MACE. Compared with those whose non-PCSK9 inhibitor lipid-lowering regimen was otherwise modified, the $\mathrm{CV}$ risk

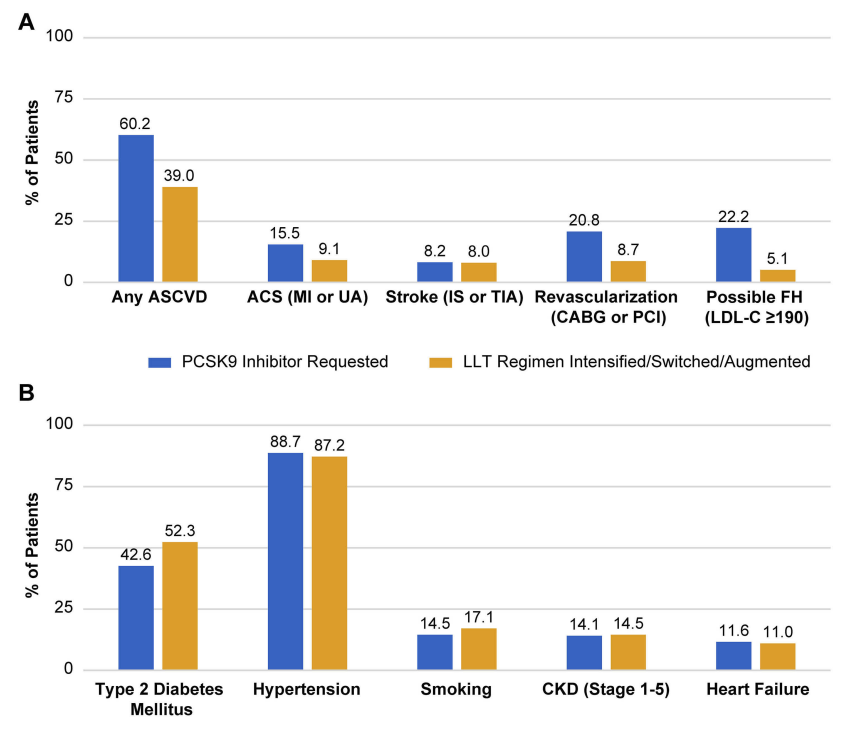

Figure 3 ASCVD diagnoses (A) and cardiovascular comorbidities and risk factors (B). Note: ASCVD diagnoses, cardiovascular comorbidities, and risk factors were measured during the 2-year pre-index period using claims from PharMetrics Plus or IQVIA medical claims database (Dx). UA was identified through in-patient claims only; other ASCVD diagnoses were identified by at least one confirmatory (ie, nonancillary) medical claim with ICD-9/ICD-I0 diagnosis codes for ASCVD conditions. "Any ASCVD" included ICD-9/ICD-10 codes for other atherosclerotic conditions such as other types of coronary atherosclerosis or other forms of chronic ischemic heart disease.

Abbreviations: ASCVD, atherosclerotic cardiovascular disease; ACS, acute coronary syndrome; CABG, coronary artery bypass graft; CKD, chronic kidney disease; $\mathrm{FH}$, familial hypercholesterolemia; ICD, International Classification of Diseases; IS, ischemic stroke; MI, myocardial infarction; LDL-C, low-density lipoprotein cholesterol; LLT, lipid-lowering therapy; $\mathrm{PCl}$, percutaneous coronary intervention; PCSK9, proprotein convertase subtilisin/kexin type 9; TIA, transient ischemic attack; UA, unstable angina.

profiles demonstrate that patients requesting access to PCSK9 inhibitor therapy had higher baseline LDL-C values and were older, with $>50 \%$ of patients meeting the high-risk criteria of being $\geq 65$ years old. In addition, patients prescribed PCSK9 inhibitors were generally more likely to have high-risk CV-related comorbidities, ASCVD diagnoses, and a recent MACE. More patients in the PCSK9 inhibitor cohort were receiving both statins and ezetimibe, suggesting greater LLT intensity in the PCSK9 inhibitor cohort. Our results are in accordance with a previous study, which found baseline LDL-C levels to be higher in patients prescribed PCSK9 inhibitor therapy compared with patients receiving other LLT. ${ }^{14}$ Interestingly, the earlier study found that higher LDL-C levels were not associated with higher PCSK9 inhibitor approval rates. Other variables that were significantly associated with approval of PCSK9 inhibitor therapy included age $>65$ years, history of ASCVD, prescription by a cardiologist or non-primary care provider, statin intolerance, longer statin duration, and noncommercial payer. $^{13}$ 


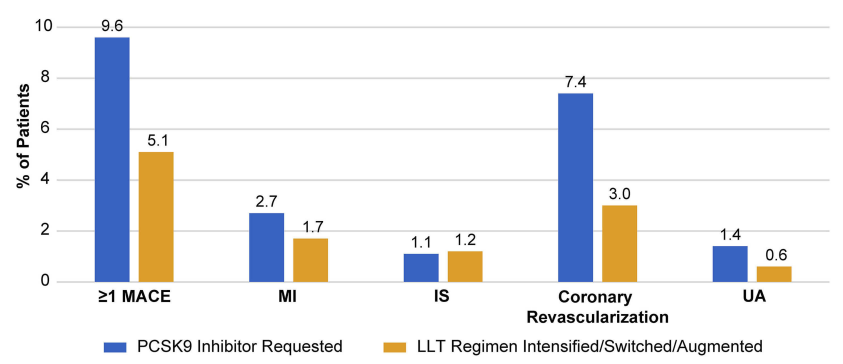

Figure 4 Cardiovascular risk: $\geq 1$ MACE during the 2-year baseline period. Note: MACE were measured during the 2-year pre-index period using $\mathrm{P}+$ or $\mathrm{Dx}$ claims (primary diagnoses from inpatient claims and any nonancillary diagnoses from the emergency department or outpatient claims)

Abbreviations: IS, ischemic stroke; LLT, lipid-lowering therapy; MACE, major adverse cardiovascular events; MI, myocardial infarction; P+, PharMetrics Plus; PCSK9, proprotein convertase subtilisin/kexin type 9; UA, unstable angina.

Although CV risk is multifactorial, LDL-C level is a critical risk factor based on evidence that lower LDL-C levels are associated with a reduced risk of $\mathrm{CV}$ events along with improved patient outcomes, including for those with LDL-C levels averaging $70 \mathrm{mg} / \mathrm{dL}$ or less. ${ }^{6,8-10,17,18}$ The ACC/AHA recommendations to extend the use of PCSK9 inhibitors in very-high-risk patients with ASCVD and monitor patient response to statin therapy and lifestyle changes were based on perspectives gleaned from the outcome trials that demonstrated that lower LDL-C is better and safe, even at very low levels. ${ }^{10,19}$ Overall, the addition of a PCSK9 inhibitor to background statin therapy has been shown to further lower LDL-C levels by $43 \%$ to $64 \%$ and also to reduce the occurrence of CV events, including MI and IS. ${ }^{8,9,20,21}$

Despite declining CVD mortality rates in the 21 st century, recent trends suggest that these rates are stabilizing and may even be on the rise because of shifting demographics, increasing prevalence of risk factors, and the lack of innovative treatments. $^{22,23}$ One recent analysis predicts that if factors such as current treatment utilization remain unchanged, CVD mortality in the US will increase by $41 \%$ in $2040 .{ }^{23}$ The authors suggest that wide utilization of innovations as impactful as the introduction of statins is necessary to stabilize and potentially further reduce CVD mortality. For patients with LDL-C $\geq 70 \mathrm{mg} / \mathrm{dL}$ despite maximally tolerated statin therapy, current guidelines recommend considering ezetimibe for patients with clinical ASCVD and adding ezetimibe before a PCSK9 inhibitor for patients with very high-risk ASCVD. ${ }^{10}$ Results from our study are consistent with other real-world studies, suggesting that augmentation with ezetimibe may not be enough for some high-risk patients to achieve LDL-C levels $<70 \mathrm{mg} / \mathrm{dL}^{24,25}$ Addition of ezetimibe to statins has shown up to $25 \%$ reduction in
LDL-C, ${ }^{26}$ and this reduction would not have been sufficient, on average, to lower the mean LDL-C from 115 to $<70 \mathrm{mg} / \mathrm{dL}$ in the group whose LLT was augmented with ezetimibe. Therefore, modifying the current non-PCSK9 inhibitor LLT without adding a PCSK9 inhibitor, in general, may not provide optimal LDL-C outcomes, particularly in patients with LDL-C elevated to the extent observed in the study cohorts.

Although MI and stroke have a similar prevalence in the US, ${ }^{1}$ we observed a lower than expected percentage of patients with a history of IS in our sample of patients whose LLT was being actively managed. This finding suggests that the IS population may be undertreated with LLT. In a recently published study of recurrent MI and IS in Medicare beneficiaries, the rate of recurrent IS during the patients' first year of survival after an event of IS (6.7\%) was similar to that of patients with recurrent MI (7.2\%), which further supports the need for aggressive intervention for secondary prevention in patients with MI or IS. ${ }^{27}$ Other important areas for future research will include investigating how the $\mathrm{CV}$ risk profile of patients in our cohort compares with the risk profiles defined in the 2018 ACC/AHA guideline, as well as assessing the impact of new guideline and the potential influence on physician prescribing practices for PCSK9 inhibitor therapy in future cohort analyses. Studies that assess the characteristics of filled vs abandoned prescriptions and impact on CV outcomes would also be of interest in the context of the changing value and access landscape for PCSK9 inhibitors.

\section{Limitations}

This was a retrospective analysis based on data linking different claims databases and is subject to limitations of conducting research using large administrative databases containing data not originally collected for research purposes. For example, it was not possible to determine whether patients were receiving maximally tolerated statin therapy or if any patients in the PCSK9 inhibitor cohort who were not receiving statins at index had been rechallenged for statin intolerance. In addition, retrospective analyses can only identify relationships; they cannot establish inference. This analysis was descriptive; thus, possible confounding factors may not be controlled for in the results. Although the analysis included both privately insured patients and Medicare beneficiaries, results should be interpreted in the context of the study sample and may not necessarily be generalizable to the US population. It was not possible to identify patients who may have received samples of PCSK9 inhibitor therapy 
during the pre-index period; therefore, only patients with LDL-C $\geq 70 \mathrm{mg} / \mathrm{dL}$ were included in the analysis. Finally, the study population was not restricted to patients with ASCVD; however, many patients not receiving statins in the PCSK9 inhibitor cohort possibly were statin intolerant and many had probable familial hypercholesterolemia, as might be expected for early adopters of PCSK 9 inhibitor therapy.

\section{Conclusions And Relevance}

Physicians are prescribing PCSK9 inhibitor therapy to patients with risk profiles consistent with recent guideline recommendations for use of PCSK9 inhibitors, including LDL-C elevations and comorbid risk factors for adverse $\mathrm{CV}$ events that suggest a higher risk profile than patients on other LLTs. Since the PCSK9 inhibitor outcomes trials and the 2018 ACC/AHA guideline support the "lowest is best" concept regarding LDL-C levels and CV risk, augmentation with ezetimibe may not be enough to achieve LDL-C levels $<70 \mathrm{mg} / \mathrm{dL}$ in many high-risk patients with ASCVD. These results may be of interest to payers and policymakers involved in devising access strategies for PCSK9 inhibitors.

\section{Abbreviations}

ACC/AHA, American College of Cardiology/American Heart Association; ASCVD, atherosclerotic cardiovascular disease; $\mathrm{CHD}$, coronary heart disease; $\mathrm{CV}$, cardiovascular; CVD, cardiovascular disease; FIA, Formulary Impact Analyzer; HIPAA, Health Insurance Portability and Accountability Act; ICD, International Classification of Diseases; IS, ischemic stroke; LDL-C, low-density lipoprotein cholesterol; LLT, lipid-lowering therapy; MACE, major adverse CV events; MI, myocardial infarction; $\mathrm{P}+$, PharMetrics Plus; PCSK9, proprotein convertase subtili$\sin /$ kexin type 9 ; UA, unstable angina.

\section{Data Sharing Statement}

Qualified researchers may request data from Amgen clinical studies. Complete details are available at the following: http://www.amgen.com/datasharing.

\section{Author Contributions}

All authors contributed to data analysis, drafting and revising the article, gave final approval of the version to be published, and agree to be accountable for all aspects of the work.

\section{Funding}

This study was sponsored by Amgen Inc. The sponsor provided funding for the study and, via employee coauthors, input into the design of the study and interpretation of the data. The sponsor reviewed the manuscript prior to submission, but the coauthors prepared, approved, and decided to submit the manuscript for publication.

\section{Disclosure}

SJB: president of Excel Medical Clinical Trials, LLC, and Preventive Cardiology, Inc; consultant/advisory board member/principal investigator for Merck, Akcea, Amgen Inc, Regeneron, Sanofi, AstraZeneca, Eli Lilly, Boehringer Ingelheim, Esperion, Gemphire, Madrigal, Novartis, Gerson Lehrman Group, Novo Nordisk and Guidepoint Global; secretary/treasurer of the $\mathrm{FH}$ Foundation; immediate past president of the American Society for Preventive Cardiology. RLW, SN, C-CC: employees of IQVIA, which was hired by Amgen Inc to conduct this study. PX, JA, CCO, CMC: employees of Amgen Inc and own Amgen Inc stock. NRD: research grants from Medtronic, Johnson \& Johnson; consulting fees from Amgen Inc, Relypsa, OPKO, scPharmaceuticals, Cytokinetics. The authors report no other conflicts of interest in this work.

\section{References}

1. Benjamin EJ, Virani SS, Callaway CW, et al. Heart disease and stroke statistics-2018 update: a report from the American Heart Association [published correction appears in Circulation. 2018;137(12):e493]. Circulation. 2018;137(12):e67-e492. doi:10.1161/CIR.0000000000 000558

2. The US Burden of Disease Collaborators. The State of US Health, 1990-2016: burden of diseases, injuries, and risk factors among US States. JAMA. 2018;319(14):1444-1472. doi:10.1001/jama.2018.0158

3. American Heart Association \& American Stroke Association. Cardiovascular Disease: A Costly Burden for America - Projections through 2035. American Heart Association website. Available from: https://healthmetrics.heart.org/wp-content/uploads/2017/10/ Cardiovascular-Disease-A-Costly-Burden.pdf. Accessed February 14, 2019.

4. Ference BA, Ginsberg HN, Graham I, et al. Low-density lipoproteins cause atherosclerotic cardiovascular disease. 1. Evidence from genetic, epidemiologic, and clinical studies. A consensus statement from the European Atherosclerosis Society Consensus Panel. Eur Heart J. 2017;38(32):2459-2472. doi:10.1093/eurheartj/ehx144

5. Goldstein JL, Brown MS. A century of cholesterol and coronaries: from plaques to genes to statins. Cell. 2015;161(1):161-172. doi:10.1016/j.cell.2015.01.036

6. Boekholdt SM, Hovingh GK, Mora S, et al. Very low levels of atherogenic lipoproteins and the risk for cardiovascular events: a meta-analysis of statin trials. J Am Coll Cardiol. 2014;64(5):485494. doi:10.1016/j.jacc.2014.02.615 
7. Wong ND, Chuang J, Zhao Y, Rosenblit PD. Residual dyslipidemia according to low-density lipoprotein cholesterol, non-high-density lipoprotein cholesterol, and apolipoprotein B among statin-treated US adults: National Health and Nutrition Examination Survey 2009-2010. J Clin Lipidol. 2015;9(4):525-532. doi:10.1016/j.jacl.2015.05.003

8. Sabatine MS, Giugliano RP, Keech AC, et al. Evolocumab and clinical outcomes in patients with cardiovascular disease. $N$ Engl $J$ Med. 2017;376(18):1713-1722. doi:10.1056/NEJMoa1615664

9. Szarek M, White HD, Schwartz GG, et al. Alirocumab reduces total nonfatal cardiovascular and fatal events: the ODYSSEY OUTCOMES trial. J Am Coll Cardiol. 2019;73(4):387-396. doi:10.1016/j.jacc.2018. 10.039

10. Grundy SM, Stone NJ, Bailey AL, et al. 2018 AHA/ACC/ AACVPR/AAPA/ABC/ACPM/ADA/AGS/APhA/ASPC/NLA/ PCNA guideline on the management of blood cholesterol: a report of the American College of Cardiology/American Heart Association task force on clinical practice guidelines. $J$ Am Coll Cardiol. 2019;73(24):e285-e359. doi:10.1016/j.jacc.2018.11.003

11. Zafrir B, Jubran A. Lipid-lowering therapy with PCSK9-inhibitors in the real-world setting: two-year experience of a regional lipid clinic. Cardiovasc Ther. 2018;36(5):e12439. doi:10.1111/1755-5922.12439

12. Baum SJ, Toth PP, Underberg JA, Jellinger P, Ross J, Wilemon K. PCSK9 inhibitor access barriers-issues and recommendations: improving the access process for patients, clinicians and payers. Clin Cardiol. 2017;40(4):243-254. doi:10.1002/clc.22713

13. Hess GP, Natarajan P, Faridi KF, Fievitz A, Valsdottir L, Yeh RW. Proprotein convertase subtilisin/kexin type 9 inhibitor therapy: payer approvals and rejections, and patient characteristics for successful prescribing. Circulation. 2017;136(23):2210-2219. doi:10.1161/ CIRCULATIONAHA.117.028430

14. Rane PB, Patel J, Harrison DJ, et al. Patient characteristics and realworld treatment patterns among early users of PCSK9 inhibitors. Am J Cardiovasc Drugs. 2018;18(2):103-108. doi:10.1007/s40256-0170246-z

15. Lewis SJ, Olufade T, Anzalone DA, Malangone-Monaco E, Evans KA, Johnston S. LDL cholesterol levels after switch from atorvastatin to rosuvastatin. Curr Med Res Opin. 2018;34(10):1717-1723. doi:10.1080/03007995.2017.1421147

16. Schuster H, Barter PJ, Stender S, et al. Effects of switching statins on achievement of lipid goals: measuring effective reductions in cholesterol using rosuvastatin therapy (MERCURY I) study. Am Heart $J$. 2004;147(4):705-713. doi:10.1016/j.ahj.2003.10.004

17. Cholesterol Treatment Trialists' (CTT) Collaboration, Baigent C, Blackwell L, et al. Efficacy and safety of more intensive lowering of LDL cholesterol: a meta-analysis of data from 170,000 participants in 26 randomised trials. Lancet. 2010;376(9753):1670-1681. doi:10. 1016/S0140-6736(10)61350-5
18. Silverman MG, Ference BA, Im K, et al. Association between lowering LDL-C and cardiovascular risk reduction among different therapeutic interventions: a systematic review and meta-analysis. JAMA. 2016;316(12):1289-1297. doi:10.1001/jama.2016.13985

19. Rubenfire M. Latest in Cardiology. American College of Cardiology website. Available from: https://www.acc.org/latest-in-cardiology/ ten-points-to-remember/2018/11/09/14/28/2018-guideline-on-manage ment-of-blood-cholesterol. Accessed January 7, 2019.

20. Sabatine MS, Giugliano RP, Wiviott SD, et al. Efficacy and safety of evolocumab in reducing lipids and cardiovascular events. $N$ Engl $J$ Med. 2015;372(16):1500-1509. doi:10.1056/NEJMoa1500858

21. Cannon CP, Cariou B, Blom D, et al. Efficacy and safety of alirocumab in high cardiovascular risk patients with inadequately controlled hypercholesterolaemia on maximally tolerated doses of statins: the ODYSSEY COMBO II randomized controlled trial. Eur Heart J. 2015;36(19):1186-1194. doi:10.1093/eurheartj/ehv028

22. Benjamin EJ, Blaha MJ, Chiuve SE, et al. Heart disease and stroke statistics-2017 update: a report from the American Heart Association [published correction appears in Circulation. 2017; 135(10):e646and Circulation. 2017;136(10):e196]. Circulation. 2017;135(10):e146e603. doi:10.1161/CIR.0000000000000485

23. Ortendahl JD, Diamant AL, Toth PP, Cherepanov D, Harmon AL, Broder MS. Protecting the gains: what changes are needed to prevent a reversal of the downward cardiovascular disease mortality trend? Clin Cardiol. 2019;42(1):47-55. doi:10.1002/clc.23097

24. Menzin J, Aggarwal J, Boatman B, et al. Ezetimibe use and LDL-C goal achievement: a retrospective database analysis of patients with clinical atherosclerotic cardiovascular disease or probable heterozygous familial hypercholesterolemia. J Manag Care Spec Pharm. 2017;23(12):1270-1276. doi:10.18553/jmcp.2017.16414

25. Chen CC, Rane PB, Hines DM, Patel J, Harrison DJ, Wade RL. Low-density lipoprotein cholesterol outcomes post-non-PCSK9i lipid-lowering therapies in atherosclerotic cardiovascular disease and probable heterozygous familial hypercholesterolemia patients. Ther Clin Risk Manag. 2018;14:2425-2435. doi:10.2147/TCRM. $\mathrm{S} 180783$

26. Lloyd-Jones DM, Morris PB, Ballantyne CM, et al. 2017 focused update of the 2016 ACC expert consensus decision pathway on the role of non-statin therapies for LDL-cholesterol lowering in the management of atherosclerotic cardiovascular disease risk: a report of the American College of Cardiology task force on expert consensus decision pathways. J Am Coll Cardiol. 2017;70(14):1785-1822. doi:10.1016/j.jacc.2017.07.745

27. Li S, Peng Y, Wang X, et al. Cardiovascular events and death after myocardial infarction or ischemic stroke in an older Medicare population. Clin Cardiol. 2019;42(3):391-399. doi:10.1002/clc.23160
Therapeutics and Clinical Risk Management

\section{Publish your work in this journal}

Therapeutics and Clinical Risk Management is an international, peerreviewed journal of clinical therapeutics and risk management, focusing on concise rapid reporting of clinical studies in all therapeutic areas, outcomes, safety, and programs for the effective, safe, and sustained use of medicines. This journal is indexed on PubMed Central, CAS,
EMBase, Scopus and the Elsevier Bibliographic databases. The manuscript management system is completely online and includes a very quick and fair peer-review system, which is all easy to use. Visit http://www.dovepress.com/testimonials.php to read real quotes from published authors. 